Methods Retrospective review of 45 randomly selected MSM attending routine HIV care (HIV+ group) OR receiving PEP (PEP group) at each of 2 London clinics during 2014/15.

Results

\begin{tabular}{lll}
\multicolumn{2}{c}{ Abstract P198 Table 1 } & RDU use in HIV+ and HIV negative MSM \\
\hline HIV+ group $(\boldsymbol{n}=\mathbf{9 0})$ & PEP group $(\boldsymbol{n}=\mathbf{8 1})$ \\
\hline Aged $<45$ & $29(32 \%)$ & $67(83 \%)$ \\
Offered SHS & $47(52 \%)$ & $73(90 \%)$ \\
Accepted SHS & $33(70 \%)$ & $68(93 \%)$ \\
Positive for STI & $8(24 \%)$ & $25(37 \%)$ \\
Asked about RDU & $40(44 \%)$ & $78(96 \%)$ \\
Ever used RDU & $18(45 \%)$ & $38(48 \%)$ \\
Used RDU in last 6 months & $8(44 \%)$ & $37(97 \%)$ \\
RDU considered problematic & $2(25 \%)$ & $14(37 \%)$ \\
\hline
\end{tabular}

The most commonly used drug was mephedrone ( $81 \%$ in PEP group vs $38 \%$ in HIV+ group) followed by crystal methamphetamine ( $54 \%$ vs $12.5 \%$ ). $2 / 81(2.4 \%)$ in the PEP group tested HIV positive within 3 months of follow up.

Discussion High levels of STIs and RDU were seen in both groups but most significantly in the PEP group. This highlights the importance of identifying RDU/chemsex in PEP patients, which may be an opportunity for intervention to reduce risk of acquisition of HIV through risky sex.

\section{P199 ANALYSIS OF TRAINING NEEDS IN A NEWLY INTEGRATED SEXUAL HEALTH SERVICE (SHS)}

Belinda Loftus*, Susan Dymock. Spectrum Community Health CIC, Yorkshire, UK

\subsection{6/sextrans-2016-052718.247}

Background The Integrated Sexual Health Services: National Service Specification 2013, includes; Patients to receive their care in a "one stop shop." Staff training should include accredited courses facilitated by BASHH and the FRSH. Key Performance Indicators that relate to the number of staff who are dual qualified.

Method Nurses were identified by their nursing role, band, original speciality and their training needs. The Band 6 (B6) nurses are the initial first training focus. Training for B6 nurses was considered as Essential; (that required to meet service specification) and Non Essential (that required in order to deliver a truly "one stop" holistic care package, or provide training to others).

Aim To identify the training needs of a newly integrated service in order to formulate a strategy which meets the training requirements of the team, whilst retaining high quality service delivery.

Conclusion Integration causes a significant training burden on SHS from a financial, organisational and workforce perspective. Meeting integration training demands is likely to cause disruption to services and staff; resulting in additional stress, increasing sickness and staff turnover rates. Training approach is based upon "quick wins" first targeting those B6 nurses with contraception and Level 2 sexual health experience. B6 Nurses were targeted first as most of their time is spent in clinic; thus improving access, and useful in upskilling others. B6 nurses considered "early adopters" were selected, as likely to have a positive effect on others.

\section{P200 EARLY DETECTION OF SEXUALLY TRANSMITTED INFECTIONS - WERE THERE MISSED OPPORTUNITIES? A QUALITATIVE STUDY IN THE UK}

${ }^{1}$ Hannah Loftus*, ${ }^{2}$ Kelly Mackenzie, ${ }^{3}$ lan Simms, ${ }^{4,3}$ Jackie Cassell. ${ }^{1}$ Sheffield Teaching Hospitals NHS Foundation Trust, Sheffield, UK; ${ }^{2}$ School of Health and Related Research, University of Sheffield, Sheffield, UK; ${ }^{3}$ Public Health England, London, UK; ${ }^{4}$ Division of Primary Care and Public Health, Brighton and Sussex Medical School, Brighton, UK

\subsection{6/sextrans-2016-052718.248}

Background/introduction The early recognition and investigation of outbreaks of sexually transmitted infections (STIs) is vital for preventing onward transmission.

Aim(s)/objectives We sought to understand the facilitators and barriers to outbreak recognition in order to improve early detection. To review the recognition and management of a series of recent outbreaks of sexually transmitted infections in the United Kingdom (UK). To formulate guidance that will enable early recognition of outbreaks.

Methods We interviewed clinicians and public health professionals who had been recently involved in identifying and managing STI outbreaks in the UK. Interviews were audio-recorded and transcribed verbatim. Transcripts were analysed using thematic analysis.

Results Ten STI outbreaks were reviewed, generally by interviewing both a clinician and public health professional. Health advisers and sexual health consultants often noticed increases in cases with smaller clinics often identifying outbreaks more quickly than larger centres through "soft" signals such as increased partner notification, contacts named multiple times or cases with similar geographical location. Sometimes changing demographics first alerted staff. In two centres, increased ceftriaxone use prompted data review. Public Health England (PHE) regional teams identified two outbreaks: one through analysis of the national dataset (GUMCAD); and one via the formal Infectious Diseases Notifications process.

Discussion/conclusion "Soft" signals, picked up in smaller clinics were less readily noticed in larger services. Although quarterly retrospective collation of electronic data by PHE currently limits their role, electronic records should be better exploited locally within services.

\section{P201 EVALUATION OF CHLAMYDIA TRACHOMATIS (CT) AND NEISSERIA GONORRHOEAE (GC) INFECTIONS IN FEMALE SEX WORKERS (FSW) ATTENDING A DEDICATED SEX WORKER CLINIC}

${ }^{1}$ Vanita Devadass, ${ }^{2}$ Kim Leverett, ${ }^{2}$ Sarah Macauley, ${ }^{1}$ Selena Singh*. ${ }^{1}$ Bart's Health NHS Trust, London, UK; ${ }^{2}$ Open Doors (City \& Hackney, Tower Hamlets \& Newham), London, UK

\subsection{6/sextrans-2016-052718.249}

Background/introduction Previous research shows that female sex workers (FSW) are at high risk of certain sexually transmitted infections (STIs), and that migrant FSW appear to be at even higher risk.

Aim(s)/objectives To evaluate the characteristics of FSW managed by our dedicated sex worker clinics who tested positive for either CT or GC, including information about sexual partners outside of work.

Methods Retrospective case note review of patients identified by the Sexual Health and HIV Activity Property Type (SHHAPT) code 'SW' who also had either CT or GC in 2012-2014. 